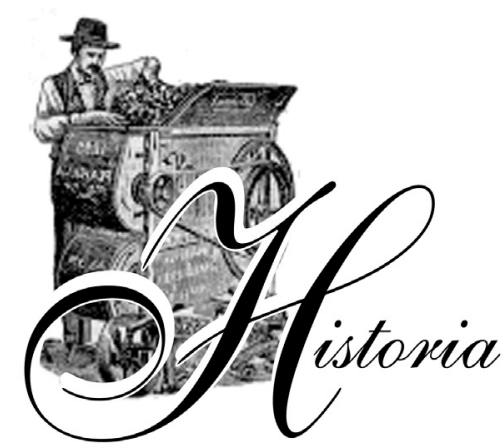

de la Ciencia en México

\title{
Técnicas e instrumentos químico-farmacéuticos en México (1849-1925)
}

\author{
Mariana Ortiz Reynoso*, Martha Díaz Flores*, Hariz Islas Flores* y Liliana \\ Schifter Aceves**
}

Recepción: 13 de octubre de 2015

Aceptación: 8 de marzo de 2016

*Universidad Autónoma del Estado de México, México.

**Universidad Autónoma Metropolitana, México.

Correos electrónicos: mortizr@uaemex.mx; marfalda08@

gmail.com; harizislas@yahoo.com.mx; liliana_schifter@

hotmail.com

Se agradecen los comentarios de los árbitros de la

revista.

\section{Introducción}

La historia de la farmacia abarca una amplia gama de temas que va desde el surgimiento de la profesión farmacéutica y la identificación de las habilidades y conocimientos peculiares a ella hasta el desarrollo de medicamentos, las formas de elaborarlos y presentarlos (Anderson, 2005).

En el mundo entero existe la profesión de farmacéutico, la cual tiene un campo de acción bien delimitado y un rol social relevante. En nuestro país, la carrera de farmacia surgió en 1833, pero los farmacéuticos desaparecieron en la segunda década del siglo xx, al transformarse en químicos farmacéuticos $y$ posteriormente en químicos farmacéuticos biólogos (QFB). En la actualidad estos profesionales tienen dominio del desarrollo, producción y control de calidad de los medicamentos, pero no está claro su papel en la farmacia comunitaria y hospitalaria. Es por ello que recientemente se han podido observar algunos cambios en el currículo; por ejemplo, en 1994 se inauguró la Licenciatura en Farmacia en la Universidad Autónoma del Estado de Hidalgo con orientación hacia la farmacia hospitalaria y clínica. Asimismo, en 1997 se abrió la Licenciatura en Farmacia en la Facultad de Farmacia de la Universidad Autónoma del Estado de Morelos, y poco a poco la oferta de este programa se ha ampliado a otras instituciones de educación superior, aunque aún son pocos los egresados a nivel 
nacional (Ortiz, 2014c). Es importante mencionar que la Organización Mundial de la Salud (OMs) reconoce la función social de este profesional y ha emitido recomendaciones sobre su perfil de egreso para atender las demandas actuales en materia de salud, en áreas estratégicas como el uso racional de los medicamentos (OMs, 2002). En este sentido, en nuestro país tenemos una deuda con las necesidades sociales en materia de salud.

Se han hecho valiosos estudios sobre las causas que llevaron a que se transformara esta profesión desde el punto de vista de las carencias ocasionadas por la falta de reglamentación y la entrada de especialidades médicas de importación (Ortiz 2014a; Aceves, 2011). Sin embargo, no se han analizado las causas desde la perspectiva de la abundancia, es decir, desde el análisis de las fortalezas de los farmacéuticos decimonónicos. Este estudio pretende ampliar los conocimientos que tenemos sobre las circunstancias que posibilitaron el cambio de farmacéuticos a químicos farmacéuticos desde el punto de vista de la labor de este profesional, estudiada a partir de los instrumentos y técnicas empleados para la elaboración de medicamentos en el siglo xix en México.

Desde la perspectiva de la historia social y cultural de la ciencia, los instrumentos científicos han permitido construir el concepto de "cultura material de la ciencia" y, junto con las técnicas químicas, han sido integrados a las nuevas líneas de investigación por considerarse fuentes importantes para reconstruir las prácticas científicas en las aulas y laboratorios (Bertomeu y Simon, 2012). Los instrumentos han puesto de manifiesto las ideas y prácticas médicas (García et al., 2005). Según Livingstone (2003), los instrumentos “son emblemáticos para el método experimental y la idea de que la naturaleza puede conocerse a través del artificio humano".
Asimismo son un indicativo del estado de la ciencia; por ello es que su estudio es de gran interés para nosotros. Por ejemplo, el refinamiento del análisis químico de los medicamentos logrado en el siglo XIX contribuyó a incrementar significativamente la seguridad de los productos farmacéuticos por sus consecuencias tanto en la exactitud como en la forma de dosificación de los fármacos. En este sentido, coincidimos con Aaron J. Ihde (2015), quien afirma que la química analítica no ha alcanzado reconocimiento suficiente debido a que a veces la aplicación de nuevas técnicas analíticas ha resultado en la generación de un conocimiento cuya importancia opaca las técnicas que hicieron posible llegar a éste (Ihde, 2015).

Junto con los instrumentos analíticos, el cambio en el mobiliario y los enseres empleados en la elaboración de los medicamentos, desde los instrumentos de manufactura oficinal hasta los equipos industriales para producción masiva, reflejan puntualmente la revolución de la práctica químico-farmacéutica de los siglos XIX y xx especialmente en Europa y Estados Unidos.

El objetivo de la investigación es conocer los instrumentos y técnicas utilizados en la docencia y en la investigación científica relativa al análisis químico de las plantas medicinales. El periodo de estudio va desde 1849, año en que fue publicado el primer texto de química escrito por un mexicano, que a decir de Aceves (2008) tiene enorme valor como fuente primaria para la historia de la enseñanza de la química: la Introducción al estudio de la química de Leopoldo Río de la Loza, y hasta 1925 cuando se publicó la última edición de la Nueva Farmacopea Mexicana publicada y editada por la Sociedad Farmacéutica Mexicana (Schifter, 2014a).

El método utilizado fue la investigación histórica que implica la localización, selección, análisis e interpretación de las fuentes documentales primarias, hemerográficas y bibliográficas. Se revisaron las siguientes fuentes primarias relacionadas con la actividad de los farmacéuticos de esta época: tesis de farmacia de la Escuela Nacional de Medicina de México, la revista científica del gremio farmacéutico llamada La Farmacia, los informes y planes de trabajo del Instituto Médico Nacional y las farmacopeas mexicanas.

Asimismo, y desde el análisis prospectivo, este trabajo intenta señalar que para asegurar el futuro de la profesión y el establecimiento de una identidad profesional poderosa para los químicos farmacéuticos biólogos y otras carreras afines es necesario analizar el papel del farmacéutico en la historia de la química en México. Con lo anterior, se pretende mostrar que la ambigüedad actual de la identidad profesional de los químicos farmacéuticos biólogos está estrechamente relacionada con las transformaciones en las prácticas de los farmacéuticos y su quehacer a finales del siglo XIX y principios del XX.

\section{Antecedentes}

En el siglo XIX mexicano la investigación químico-farmacéutica se llevó a cabo en dos recintos principalmente: la Escuela Nacional de Medicina (ENM) y el Instituto Médico Nacional (IMN).

\section{1. La Escuela Nacional de Medicina y la Sociedad Farmacéutica Mexicana}

La carrera de farmacia comenzó a impartirse a partir de la fundación de la ENM en 1833 con una única cátedra: Farmacia teórico-práctica (Aceves, 1997). A partir de 1867 se impartieron dos cátedras más: Historia Natural de las Drogas y Análisis Químico. Los textos de consulta, predominantemente europeos, se eligieron en torno a estas asignaturas. Más adelante, se 
aumentó un segundo curso de Análisis Químico. La tabla 1 muestra el plan de estudios de farmacia vigente durante el último tercio del siglo.

Para el curso de Análisis Químico se utilizaban principalmente dos textos: el de Fresenius y el Gerhardt y Chancel. La tabla 2 resume los temas revisados en cada uno de ellos durante las clases.

Como se desprende de la tabla 2, a lo largo de los dos cursos téorico-prácticos de Análisis Químico impartidos en la carrera de Farmacia, los estudiantes adquirían los conocimientos necesa-

Tabla 1.

Plan de estudios de Farmacia de 1893, Escuela Nacional de Medicina. ${ }^{*}$

\begin{tabular}{|c|c|c|}
\hline Año & Nombre de la cátedra & Nombre del profesor y del texto \\
\hline \multirow{2}{*}{$1^{\circ}$} & Farmacia teórico-práctica & José Donaciano Morales (1877-1920) \\
\hline & & a) Andouard, Farmacia elemental \\
\hline \multirow{7}{*}{$2^{\circ}$} & & Juan Manuel Noriega (1901-1924) \\
\hline & Historia natural de las drogas simples & a) Planchon y Colin, Drogas simples \\
\hline & & b) Herland, Medicamentos minerales \\
\hline & Primer curso de Análisis Químico & Víctor Lucio Ortega (1884-1918) \\
\hline & & a) Gerhart y Chancel, Análisis \\
\hline & & b) K. R. Fresenuis, Análisis \\
\hline & & c) R. D. Silva, Análisis \\
\hline \multirow[t]{4}{*}{$3^{\circ}$} & Segundo curso de Análisis Químico & Víctor Lucio Ortega (1884-1918) \\
\hline & & a) Gerhart y Chancel, Análisis \\
\hline & & b) K. R. Fresenuis, Análisis \\
\hline & & c) R. D. Silva, Análisis \\
\hline
\end{tabular}

Nota: *Los años corresponden a su labor de profesor titular propietario de la cátedra. Fuente: elaboración propia.

Tabla 2. Temas estudiados en los libros de texto de las cátedras de Análisis Químico.

\begin{tabular}{|c|c|}
\hline Nombre del autor y del texto & Temas revisados en el texto \\
\hline \multirow[t]{3}{*}{ Ch. Gerdhardt y G. Chancel } & Aparatos de análisis, operaciones generales \\
\hline & Análisis mineral \\
\hline & Análisis elemental sustancias orgânicas \\
\hline \multirow[t]{3}{*}{ Précis d'Analyse chimique qualitative } & Métodos especiales para análisis de aguas minerales, \\
\hline & cenizas, tierras arables \\
\hline & Exposición de los cálculos \\
\hline \multirow[t]{3}{*}{ K. R. Fresenius } & Reactivos, aparatos y manipulaciones \\
\hline & Análisis general de sustancias simples y compuestas \\
\hline & (solubles e insolubles) \\
\hline \multirow[t]{8}{*}{ Traité d'Analyse chimique quantitative } & Análisis especiales (aguas minerales, cenizas, silicatos, \\
\hline & cianurados insolubles en agua, detección de sustancias \\
\hline & inorgánica en presencia de materia orgánica \\
\hline & Reacciones y métodos de separación cuerpos inorgáni- \\
\hline & cos raros \\
\hline & Reacciones y separación de compuestos orgánicos (alca- \\
\hline & loides, ácidos orgánicos) \\
\hline & Detección de venenos \\
\hline
\end{tabular}

mente a la química analítica, la cual tuvo un papel de liderazgo en los avances de este campo de estudios que sin embargo no está citada en los trabajos de la ENM o del IMN (Ihde, 2015). Por otro lado, el texto Précis d'analyse chimique qualitative de Ch. Gerhardt y G. Chancel, que tuvo varias publicaciones en la segunda mitad del siglo XIX, también fue uno de los libros icónicos para la implementación de técnicas del análisis químico (De los Ríos, 2011).

Durante el siglo XIX la plantilla de profesores de la ENM estuvo conformada por Leopoldo Río de la Loza (18071876), José María Vargas (1788-1875), Gumesindo Mendoza (1834-1884), Alfonso Herrera (1838-1901), J. Manuel Lasso de la Vega (¿?-1906) y Alejandro Uribe (¿?), además de los citados en la tabla 1. La gran mayoría pertenecían a la Sociedad Farmacéutica Mexicana (SFM) fundada en 1871 por Leopoldo Río de la Loza (Aceves, 2011). Entre sus funciones, la SFM estaba encargada de la publicación de la Nueva Farmacopea Mexicana que alcanzó cinco ediciones entre 1874 y 1925 (Schifter, 2014b). En estos textos se pone de manifiesto el interés de los farmacéuticos mexicanos por difundir la utilidad terapéutica de las plantas medicinales nacionales dando continuidad a los trabajos de Cervantes (cuyo manuscrito original data de 1791, pero que fue publicado en la revista El Estudio en 1889) y de la Cal (1832), y situar a la materia médica vegetal de México como protagonista en todas sus ediciones (Aceves, 1995; Huerta, 2000, Schifter, 2014b).

En la ENM, los alumnos de la carrera de farmacia realizaron más de cien tesis prácticas durante el siglo XIX, gracias a las cuales se conocen las líneas de investigación, así como las técnicas empleadas para el análisis químico de las plantas (Ortiz, 2002). Como ya se ha mencionado, la principal línea de investigación farmacéutica durante el 
siglo XIX fue el estudio sistemático de las especies vegetales mediante el empleo de las técnicas analíticas más populares en su tiempo, conocidas colectivamente como Método general de análisis. Los conocimientos necesarios para realizar los análisis eran adquiridos por los alumnos de la carrera de Farmacia en la cátedra de Análisis Químico impartida primero por Leopoldo Río de la Loza y después (y durante más de 20 años) por Víctor Lucio Ortega.

El hallazgo de un alcaloide, entendido en esa época como una molécula con actividad biológica que reaccionaba con los ácidos, era de sumo interés para los farmacéuticos debido a sus posibles aplicaciones terapéuticas futuras. La importancia de la búsqueda de alcaloides en las plantas medicinales nacionales para la ENM está representada en las numerosas tesis de farmacia dedicadas al tema (52.6\%). Si se excluyen tópicos como legislación farmacéutica, análisis de agua o salud pública y únicamente se cuantifican las tesis sobre plantas o animales con valor terapéutico, este dato se eleva a casi al $71 \%$ (Ortiz, 2002).

La contribución de los académicos investigadores de la ENM y sus alumnos al estudio de las plantas medicinales fue muy destacada. Los resultados obtenidos a partir de las tesis mencionadas enriquecieron las páginas de las Farmacopeas Mexicanas de 1896,1904 y 1925, donde se incluyeron monografías para las plantas medicinales estudiadas en ese recinto y sus aplicaciones terapéuticas (Schifter, 2014b).

\section{2. El Instituto Médico Nacional, un espacio para el desarrollo del análisis químico}

En 1888 se creó una institución clave para la práctica del análisis químico que se desarrolló a finales del siglo XIX y principios del xx en nuestro país: el Instituto Médico Nacional (IMN). Al momento de su fundación, el objetivo del IMN fue el estudio de la fauna y flora y sus aplicaciones útiles para la medicina y la industria. También se propuso el estudio de la geografía médica y la climatología del país de ahí que desde su concepción naciera con una vocación social. El primer presidente fue el naturalista Fernando Altamirano (1848-1908), quien estuvo más de diez años al frente del Instituto. Dentro del plantel, destacan algunos notables farmacéuticos decimonónicos, entre ellos: Donaciano Morales (1850-1929), Francisco Río de la Loza (1856-1890) y Mariano Lozano y Castro (¿?). Posteriormente, también formaron parte del plantel farmacéuticos de generaciones posteriores que también fueron directores de la Facultad de Química, como Roberto Medellín (de 1920 a 1921 y de 1929 a 1931), Adolfo P. Castañares (de 1918 a 1919) y Ricardo Caturegli (de 1924-1927 y luego en 1944 como interino).

En su origen el establecimiento estuvo conformado por cinco secciones: Historia Natural, Fisiología Experimental, Química Analítica, Terapéutica Experimental y Geografía y Climatología médica. En 1903 se creó el Departamento de Química Industrial que pondría especial énfasis en los productos farmacéuticos y su posible fabricación en escala industrial.

A lo largo de 26 años de investigación, el IMN publicó gran cantidad de información relativa a la flora endémica del territorio que había sido desconocida o poco estudiada por las ciencias químicas y la farmacología internacionales (Hinke, 2012; Azuela, 1994). El análisis químico relacionado con la identificación y aislamiento de los compuestos con actividad terapéutica se realizaba en la sección de Química Analítica conformada prácticamente en su totalidad por farmacéuticos egresados de la ENM.
Estos farmacéuticos eran especialistas en análisis químico y desarrollaban sus actividades en diversas instituciones de forma simultánea. En efecto, la mayoría eran docentes de la ENM y analistas en los laboratorios del Consejo Superior de Salubridad y el IMN que conformaban una red académica-profesional de expertos en el análisis químico asociada al estudio de compuestos orgánicos (Aceves y Martínez, 2010).

Para 1905 en el herbario de plantas de México en poder del IMN figuraban cerca de 10000 ejemplares y más de 2 000 aparecían en el catálogo, incluyendo sinonimia vulgar, aplicaciones, ilustraciones y fotografías de cada una de ellas (Morales, 2014). El instituto fue clausurado en 1915, pero su legado quedó plasmado en El Estudio y los Anales del Instituto Médico Nacional, así como en sus textos la Farmacología Nacional (1913) y los cinco volúmenes de los Datos para la Materia Médica Mexicana. Hay que mencionar sus aportaciones a las tres últimas ediciones de la Farmacopea Mexicana (1896, 1904, 1925), especialmente a la edición de 1925, a decir de Schifter (2014b: 48): "es la obra más completa, la que consigue reunir el conocimiento científico acerca de las especies naturales vegetales propias del suelo mexicano, los productos químicos más utilizados, y las preparaciones emanadas de la práctica farmacéutica nacional".

El trabajo que el IMN realizó en materia de botánica, química, geografía y farmacología resulta portentoso e invaluable en términos de la historia de la materia médica mexicana. La convergencia de intereses científicos con la ENM y la SFM hizo posible el estudio sistemático de la flora nacional. Estos tres órganos conformaron la élite farmacéutica del siglo XIX mexicano, cuya vasta producción científica es testimonio de la calidad de su trabajo, de donde destaca el análisis químico. 
En la segunda década del siglo xx ocurrió la transformación de la carrera de Farmacia a la de Químico Farmacéutico. El detonante de esta metamorfosis fue precisamente el dominio de las técnicas de análisis químico por parte de los farmacéuticos, aunque existen otros factores que también propiciaron este cambio de identidad: la entrada de los medicamentos de patente de importación, la carencia de un marco legal en materia sanitaria que respaldara la relevancia de su rol social y su disgusto con los médicos, con quienes llevaban compartiendo espacios desde la creación de la ENM y frente a los cuales se les consideraba como profesionales de segunda categoría (Ortiz et al., 2008).

La consecuencia de su nueva identidad fue su inserción en la Escuela Nacional de Industrias Químicas, antede la Universidad Nacional Autónoma de México (Martínez et al., 2007). Esto explica por qué en nuestro país casi la totalidad de los programas de farmacia se imparten en las facultades de química. La identidad del farmacéutico está íntimamente vinculada a la química, pues cesora de la actual Facultad de Química

más de 90\% de los programas académicos del área farmacéutica del país son de químico farmacéutico biólogo (Giral, 2015).

\section{Resultados}

\section{1. Los métodos experimentales de investigación químico- farmacéutica}

Los tesistas de la ENM y los analistas de la sección de Química Analítica del IMN analizaban las plantas de la misma forma: por las vías seca (análisis inorgánico) y húmeda (análisis orgánico). Para conocer la composición inorgánica se calcinaban cerca de 10 g de droga (planta) pulverizada y se pesaba el residuo inorgánico. Para hallar la composición orgánica se efectuaba la extracción y separación de las "familias" de compuestos. La técnica consistía en tomar una muestra de aproximadamente $100 \mathrm{~g}$ de droga pulverizada y efectuar maceraciones subsecuentes en distintos disolventes orgánicos, como se muestra en la figura 1. Con ello se buscaba la separación y extracción de los principios activos según sus solubilidades rela-

Figura 1. Esquema del Método general de análisis de las especies vegetales referido en las tesis de farmacia de la ENM y los Anales del IMN.

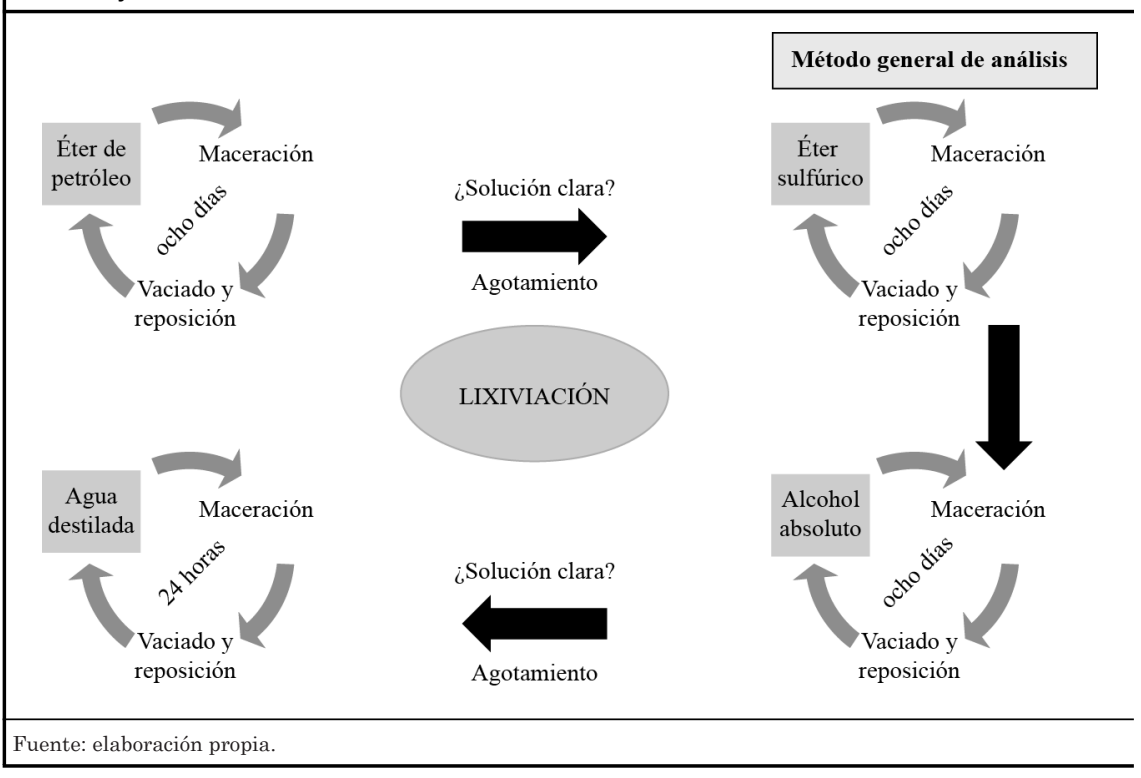

tivas en distintos disolventes.

La maceración en éter de petróleo extraía grasas, esencias, ceras y resinas, del éter sulfúrico se obtenían ácidos, glucósidos, alcaloides y resinas, en la fracción de alcohol absoluto se aislaban resinas, principios amargos, glucósidos, alcaloides, taninos, azúcares, materias colorantes y sales minerales y, por último, en el agua destilada se quedaban péptidos, azúcares, ácidos, hidratos de carbono, materias albuminoides y saponinas. Como puede advertirse de la figura 1, cada análisis tardaba más de tres semanas en llevarse a cabo, solamente contabilizando los agotamientos en los distintos disolventes. A este tiempo se sumaba el dedicado al resto de la experimentación química, pero también el invertido en la herborización o recolección de las especies vegetales que se hacía frecuentemente en zonas cercanas a la Ciudad de México, como Tacubaya o San Ángel, por mencionar sólo dos sitios.

Luego de las extracciones se acidulaba o alcalinizaba ligeramente el medio acuoso para procurar encontrar a los alcaloides en su estado de base libre o sal, según convenía para su aislamiento. Esto se hacía con cada una de las fracciones obtenidas anteriormente. Para identificar con precisión a los alcaloides, los analistas recurrían al reactivo de Dragendorff -formado por una mezcla de yoduro de potasio y bismuto, y que hoy en día sigue siendo útil para la identificación rápida de alcaloides en una muestra-. De hecho en las tesis de farmacia, los estudiantes se refieren a esta etapa del proceso como la marcha de Dragendorff.

Otro método común para la detección de alcaloides era el de Stas, descrito por Ricardo Pérez en su Estudio sobre la falsa belladona (Pérez, 1885). El método consistía en detectar el alcaloide con reactivos de Bouchardat (solución acuosa de yodo/ioduro de potasio) y Mayer (solución acuosa de cloruro de 
mercurio/ioduro de potasio), lo cuales continúan empleándose hoy en día para la identificación cualitativa y rápida de alcaloides de cualquier estructura. ${ }^{1}$

Estas series de operaciones de separación y destilación eran conocidas genéricamente como Método general de análisis por los investigadores de la ENM y el IMN y estaban reportadas en la literatura europea. Los farmacéuticos mexicanos aplicaban este método a las plantas nacionales con ligeras modificaciones. El resultado era la clasificación de los componentes obtenidos a partir de las especies vegetales en ácidos, bases, sales, gomas, resinas, colorantes y principios activos, los cuales podían ser alcaloides, glucósidos o aceites esenciales.

Algunas veces se efectuaba también la caracterización fisicoquímica de las moléculas encontradas, lo cual significaba medir los puntos de fusión y ebullición, la rotación de la luz y determinar los productos de ciertas reacciones químicas con ácidos, minerales o licor de Fehling.

La posibilidad de tener el fármaco en un estado concentrado y relativamente puro revolucionó las prácticas médicas y farmacéuticas en el mundo y además acentuó la clasificación-otrora tácita-de los medicamentos en dos grupos: los peligrosos y los de bajo riesgo (Ortiz, 2014b).

A partir de las moléculas aisladas, los investigadores del IMN desarrollaron fórmulas magistrales, pero también polvos, tabletas e inclusive cápsulas para dosificar adecuadamente los principios activos.

\section{2. Instrumentos de análisis y operaciones farmacéuticas descritas en los textos de Farmacia de la ENM} En México el material de vidrio utilizado en los recintos académicos, de investigación o industriales estaba conformado principalmente por frascos, matraces, sifones, refrigerantes, agitadores, gasógenos, lámparas, termómetros, sopletes, jeringas y recipientes varios de capacidad pequeña. Como apunta Río de la Loza (2008: 66): "en la industria los hay de vidrio hasta de veinte litros, siendo aún mayores los de barro llamados porrones y los metálicos". La capacidad volumétrica permite ponderar la incipiente escala de la producción industrial mexicana.

En el IMN, para llevar a cabo el análisis químico de las plantas, había que usar primero balanzas y estufas u hornos de secado para obtener la muestra en cantidades exactas. Luego debían echarse a andar los lixiviadores o digestores y destiladores en los que se dejaba macerar la muestra por varios días, como hemos explicado antes.

Sobre los equipos para lixiviación, Alfonso Luis Herrera (1889: 18) recomienda "valerse del aparato de Guérin ó el de Payen para impedir la pérdida de disolvente, [y] acelerar la operación". También señala que "el lixiviador continuo de Damoiseau es más sencillo y menos costoso que aquellos" cuando se trata de separar los solutos disueltos del sulfato de cal. Estas descripciones muestran que si bien los farmacéuticos emulan los instrumentos y equipos europeos, también abren la posibilidad de modificarlos para adecuarlos a los materiales y necesidades nacionales.

La aplicación del microscopio en la química era la de caracterizar físicamente la forma cristalina y el tamaño de una entidad química aislada. El estudiante Lozano y Castro, quien posteriormente se desempeñaría como químico analista de la segunda sección del IMN describe en su tesis que el alcaloide psoralina tiene forma de largas agujas prismáticas de $0.005 \mathrm{~m}$ de longitud aproximadamente, que sólo se perciben con ayuda de este instrumento (Lozano y Castro, 1884, citado en Hinke, 2012).

La Farmacopea Mexicana de 1846 es la única de las cinco ediciones que refiere una escueta, pero efectiva lista de utensilios para la elaboración de preparados farmacéuticos, en ella se encuentran los aparatos para destilación como los alambiques de cobre estañado, las retortas y los aparatos de desalojamiento y evaporación. La lista incluye a las balanzas para conocer la masa de las sustancias, los morteros para moler y los termómetros para medir la temperatura. Asimismo, incluye material de vidrio (sifones, probetas) y de porcelana (cápsulas, crisoles) básico para un laboratorio (Academia Farmacéutica de la Capital de la República, 1846).

Las ediciones posteriores de esta obra obvian los utensilios necesarios para elaborar los medicamentos. Sin embargo, se puede inferir su uso a partir de una fórmula dada; por ejemplo, el aparato para destilación se refiere implícitamente en la monografía de la yerba santa (Piper sanctum). "El Sr. Cal dice que por la destilación de esta planta se obtiene un aceite esencial más pesado que el agua y semejante al de sasafrás". (Sociedad Farmacéutica Mexicana, 1874: 219).

La Farmacopea Mexicana de 1874 incluye 13 tablas en los prolegómenos de la obra en las que se indican las equivalencias en unidades de masa (onza, quintal, arroba, grano, libra y adarme vs. sistema métrico decimal), grados de temperatura (Reaumur, Fahrenheit vs. centígrados), grados de densidad (Cartier vs. grados centesimales y grados del aerómetro de Baumé vs. densidades

1. Otros reactivos reportados en las tesis de Farmacia de la ENM son azul de Prusia, licor de Fehling, ácidos clorhídrico, sulfúrico y nítrico, bicromato de potasa percloruro de fierro (sic), bicloruro de mercurio, glicerina, alcohol, éter, amoniaco, cloruro de bario subacetato de plomo y los reactivos de Mayer, Ermann, Scheibler, Froehde y Nessler. A partir de estas sustancias puede verse que las operaciones realizadas eran propias del análisis químico y no de la farmacia galénica, la cual consiste en la preparación de fórmulas medicinales (salvo la glicerina). 

de 1904 y 1925 amplían notablemente la información contenida en dichos prolegómenos.

\section{3. Los instrumentos para el análisis químico de plantas medicinales utilizado en el IMN}

El trabajo del IMN (Instituto Médico

Nacional) se caracterizó por identificar los principios activos en las especies vegetales medicinales más comunes del país y determinar su posible aplicación terapéutica. de líquidos varios). Las farmacopeas

Todas las drogas que se han estudiado han sido tomadas de aquellas que son más usuales entre los herbolarios ó en las Farmacias. La mayor parte de ellas corresponden á las que cita la Farmacopéa Mexicana, que contiene próximamente 500 de las cuales ha estudiado yá el Instituto más de 100. Hemos notado al hacer estos estudios que las plantas que actualmente usa el vulgo son, casi todas, de las que usaban también los antiguos mexicanos y que nos fueron transmitidas primero por el Dr. Hernandez, después por los autores de la

\section{Figura 2. Aparato de baño maría del IMN.}

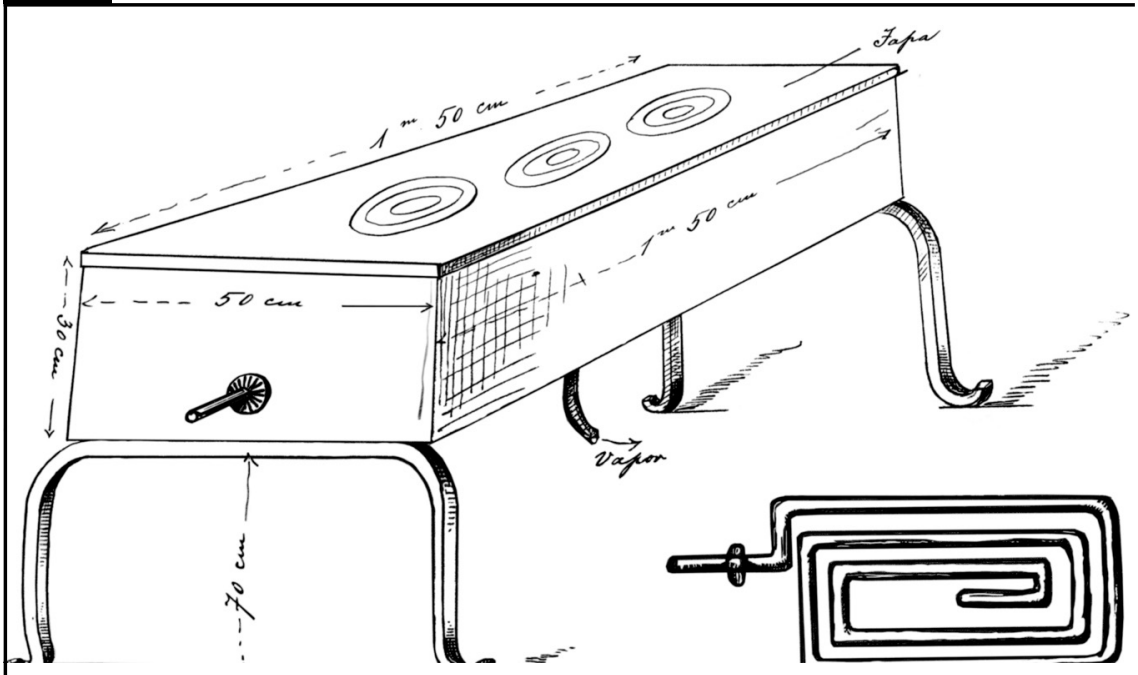

Fuente: Altamirano, 1903

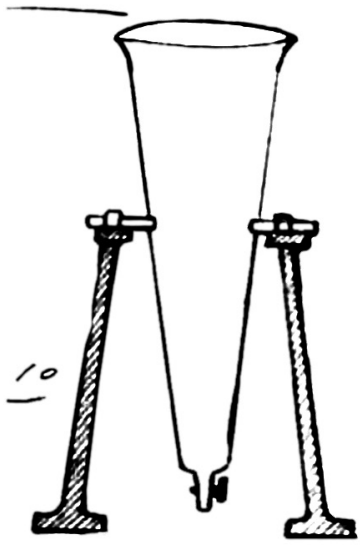

Fuente: Altamirano, 1903
Flora Mexicana [Mociño y Sessé] y en seguida por las Sociedades científicas, especialmente por la Farmacéutica hasta la época del Instituto Médico (Altamirano, 1905: 3).

Como ya hemos mencionado, el método de análisis utilizado no varió sustancialmente del implementado en la ENM si bien en los primeros años del siglo xx se hicieron algunas optimizaciones a los procesos de análisis, sobre todo en cuanto a la reducción en los tiempos de extracción de las moléculas activas. El material y los instrumentos para el análisis general utilizados por los científicos del IMN eran balanzas, morteros de porcelana y hierro, tamices, cilindros de vidrio graduados, vasos, frascos, embudos, matraces, sifones, pipetas, varillas, desecadores, termómetros, estufas de hierro con doble pared y baños maría. Los análisis se efectuaban en equipos de 0.5 a 21 de capacidad aproximadamente. Para las filtraciones se utilizaba papel filtro de la marca Schleicher \& Schuell de los números 595 y 589 .

La figura 2 muestra el dibujo de un aparato de baño maría por el cual se hacía pasar vapor como sistema de calentamiento. Las dimensiones del equipo indicadas en el gráfico son: 1.50 $\mathrm{m} \times 50 \mathrm{~cm} \times 70 \mathrm{~cm}$.

La manipulación preliminar de las drogas se llevaba a cabo mediante el uso de molinos, cilindros de loza o barro para macerar, tamices con malla de lata y morteros de hierro. Para la fabricación de esencias eran necesarios aparatos para la destilación con vapor, como una retorta de lata o porcelana, un condensador de Liebig y los frascos florentinos. Para obtener los aceites esenciales se recurría a una prensa. Para la extracción de principios activos se ocupaban coladores de lata con soportes de madera como el que se muestra en la figura 3. 
Ahí dentro se colocaban las raíces, hojas u otras partes de las plantas a analizar.

La extracción de alcaloides se efectuaba con una retorta de cobre forrada de estaño, provista con un sistema de calentamiento por vapor, dentro de la cual se vertía alcohol, tricloruro de metilo u otros solventes. La retorta se unía a una vasija en la que se colocaba la materia vegetal previamente pulverizada y macerada. Ambos equipos se conectaban a su vez con un condensador, dentro del cual había un serpentín de estaño rodeado de agua fría por el que viajaban los vapores producto de la destilación.

Para la destilación de alcaloides y glucósidos los instrumentos y equipos necesarios eran unos coladores de lata con soportes de madera, el aparato para la extracción de alcaloides, un alambique de cobre, un agitador, botellas de loza y cápsulas de hierro esmaltado, loza o porcelana. Por medio de la operación de destilación se preparaban formas farmacéuticas como hidrolatos, aguas destiladas, alcoholatos y aceites esenciales.

Para recobrar los disolventes por destilación se echaba mano de un alambique de cobre forrado con estaño o lata calentado con vapor. Para evaporar y sacar los últimos residuos de disolventes se usaba una cápsula de hierro esmaltado, también calentada por vapor.

Para sacudir (agitar) los extractos con cloroformo o éter sulfúrico, a decir de Altamirano (1905: 6), "la forma más práctica es aquella de una mantequera inglesa movida por máquina de vapor." La mantequera consistía en un recipiente montado en unos soportes, provisto con una polea que lo hacía girar sobre el eje horizontal. Dentro del agitador se colocaban los frascos embalados con paja o papel. Otros materiales usados para este fin eran embudos de separación, cilindros de vidrio con tapas esmeriladas para cristalización, desecadores al vacío y aparatos para filtrar con trompa (estos últimos indispensables para efectuar el aislamiento de alcaloides).

\section{4. Métodos de análisis químico de medicamentos reportados en los textos de farmacia de la época} Hasta aquí hemos visto las operaciones e instrumentos utilizados para la fabricación de medicamentos, particularmente para el aislamiento de principios activos de las especies vegetales. Ahora veremos algunos métodos de análisis de medicamentos reportados en los textos científicos de la época, los cuales son menos frecuentes en la literatura; de hecho, durante el siglo XIX una de las quejas de los farmacéuticos era que no tenían forma de verificar la composición y calidad de los medicamentos, especialmente los de patente.

La estandarización de los medicamentos fue un tema de sumo interés para los farmacéuticos. En particular, los métodos para evaluar medicamentos de empleo delicado como el opio resultaban de gran utilidad. Este agente terapéutico es en realidad una mezcla de muchas sustancias cuya cantidad de alcaloide puro (por ejemplo morfina o codeína) varía de región a región y de cosecha a cosecha.

Por ello, en los textos farmacéuticos decimonónicos pueden encontrarse técnicas para cuantificar los principios activos de distintas drogas (plantas, semillas o preparados intermedios para elaboración de medicamentos). Pontes Dorvault, en su famoso formulario del siglo XIX, sugiere un método para la evaluación de las cápsulas (semillas) de adormidera y la consecuente cuantificación de morfina $\left(\mathrm{C}^{34} \mathrm{H}^{19} \mathrm{NO}^{6}\right.$ ) en la flor (Dourvault, 1867). ${ }^{2}$ Las técnicas para extraer y cuantificar los alcaloides del opio no perdieron relevancia durante el primer cuarto del siglo xx.
La revista La Farmacia, órgano de divulgación de la comunidad farmacéutica, publicó en 1921 un método para extraer morfina de la flor de amapola. Los autores recomiendan digerir y lixiviar al Soxhlet con alcohol de $95^{\circ}$ la parte de la flor en cuestión. El filtrado, desecado con acetato de plomo, se somete a un examen colorimétrico que puede ser cualquiera de los dos siguientes: a) reducción del ácido yódico con producción de coloración amarilla, sensibilizada por adición de amoníaco con límite de cuantificación de $1 / 5500$ y $b$ ) coloración azulada del alcaloide con el reactivo sulfoformulado, con límite de cuantificación de 1/14000 (Heiduschka y Falul, 1921).

La misma revista publicó un método para la dosificación de la codeína, consistente en la extracción del alcaloide del opio seco con ácido acético y extracto de Saturno (mercurio), en el que se forma la sal clorhidrato que luego es cristalizada (Annet, 1921). En la figura 4 se muestra este procedimiento en un diagrama de flujo.

Otro punto de interés farmacéutico que cabe destacar fue la discusión sobre los métodos de esterilización necesarios para los productos parenterales (inyectables), nuevamente con especial interés en los medicamentos de empleo delicado, como es el caso de los preparados de cocaína inyectables. Este tema fue objeto de varios artículos científicos, ya que suponía un reto técnico, pues la esterilización de la cocaína por medio de calor inactiva la molécula provocaba la pérdida de su efecto terapéutico. Varios números de la revista La Farmacia publicados a

2. Si se compara esta fórmula química con la actualmente aceptada para la morfina, se nota enseguida que difiere en el número de carbonos y oxígenos exactamente en un múltiplo de dos. El error es consecuencia del cálculo de los equivalentes de carbono y oxígeno propio de la época. 
principios del siglo xx estudian la reacción química por la cual se produce la inactivación de la cocaína (o su clorhidrato) por calor (Pérez, 1905).

\section{Discusión de resultados}

El dominio del análisis químico de los farmacéuticos decimonónicos y su aplicación a las "drogas" de origen vegetal les permitieron la caracterización química de los compuestos contenidos en las plantas medicinales y el descubrimiento de numerosos habían utilizado desde tiempo atrás en la medicina tradicional.

La disponibilidad del fármaco concentrado $-\mathrm{y}$ no disperso en una materia en bruto- permitió mejorar hasta niveles nunca antes vistos su estandarización y dosificación, así como la intensidad de sus efectos. Asimismo, generó medicamentos más potentes que los conocidos hasta ese momento. Si bien el desarrollo de la química farmacéutica industrial se había manifestado hacía medio siglo en Alemania, Inglaterra y Estados Unidos (Friedrich, 1996), en México este proceso de industrialización se dio de forma mucho más discreta y tardía a mediados del siglo xx. Los resultados obtenidos a partir de las principios activos en plantas que se

plantas endémicas y sus principios activos no pudieron llevarse a la escala industrial.

Sin embargo, en México, el conocimiento de los instrumentos y métodos de análisis químico acumulado por los farmacéuticos del siglo xIx les permitió sentar las bases para la institucionalización de la enseñanza de la química alcanzada en la segunda década del siglo xx. En sus inicios fueron los farmacéuticos quienes llevaron la tutela de la Facultad de Química y los campos emergentes relacionados con la química fueron gradualmente incluidos en las nuevas carreras hasta convertirse en las que conocemos en la actualidad. La institucionalización de la química en nuestro país estuvo marcada en el siglo XIx por la cercanía de los farmacéuticos con el análisis químico. El análisis de este proceso nos proporciona los elementos necesarios para entender la identidad histórica de los QFB (Químico Farmacéutico Biólogo) quienes hoy en día se relacionan más con la química que con la farmacia; de hecho, en el argot de ambientes profesionales como la industria farmacéutica o los hospitales son llamados simplemente químicos. Esta denominación se gestó sin duda durante la segunda mitad del siglo XIX en México.

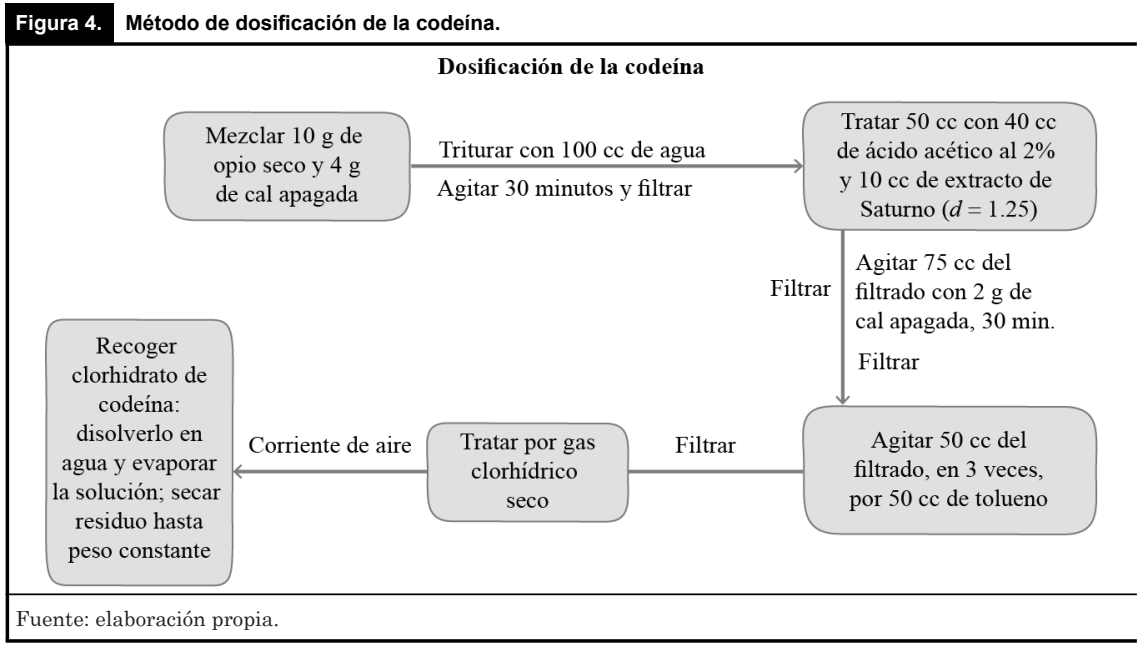

\section{Conclusiones}

El arribo de la crisis de la farmacia como profesión durante la primera mitad del siglo xix (Aceves, 2011) provocó la movilización de los farmacéuticos de la ENM, el IMN y la SFM para asegurar nuevos espacios de desarrollo profesional. Su estrategia se centró en el análisis químico de las especies vegetales del país para identificar sus principios activos y así poder establecer un nueva "terapéutica nacional" independiente de las necesidades del extranjero (Schifter, 2014b). Sus trabajos permitieron clasificar los componentes de la materia médica tradicional en ácidos, bases, sales, gomas, resinas, colorantes y principios activos (especialmente alcaloides).

Las operaciones farmacéuticas asociadas al análisis químico durante el periodo de estudio eran la trituración de las plantas, seguida de las maceraciones con distintos disolventes y el tratamiento ácido/básico de cada fracción obtenida para finalmente identificarlas como alcaloides o glucósidos. En suma, el énfasis metodológico e instrumental de la farmacia del siglo XIX mexicano se encuentra en la extracción y aislamiento de los principios activos provenientes de la flora nacional a fin de a generar productos que pudiesen competir con los de importación.

El estudio de las fuentes consultadas permitió comprobar que los farmacéuticos mexicanos conocían y utilizaban los equipos europeos propios del análisis químico durante el siglo XIX, e inclusive discutieron la idoneidad de cada uno de ellos y sugirieron algunas adecuaciones.

El cambio en la producción y control de insumos farmacéuticos sucedida en las primeras décadas del siglo xx se evidencia a partir de dos indicadores aquí revisados: a) por un lado el 
creciente interés en la evaluación de la calidad de los medicamentos mediante la cuantificación de los principios activos y $b$ ) por otro lado, la difusión de métodos farmacéuticos emergentes como la obtención de preparados estériles de uso parenteral.

\section{Prospectiva}

En 2015 se reavivó la discusión a nivel nacional acerca de la posibilidad de homologar los planes de estudio de farmacia. Existe una hipernominalidad para designar a los profesionales de la farmacia: químico farmacéutico biólogo, químico farmacobiólogo, farmacéutico, ingeniero farmacéutico, químico farmacéutico industrial, químico biólogo parasitólogo, químico biotecnólogo, etcétera. Hasta ahora, el debate gira no sólo en torno a las competencias que debe tener este profesionista, sino hacia la pertinencia del título de químico farmacéutico biólogo o la posibilidad de retomar nuevamente el título de farmacéutico imperante en el resto del mundo. La toma de esta decisión sobre el futuro de la profesión farmacéutica no puede estar aislada del entendimiento de la historia de esta carrera. Entre los atributos relevantes de los farmacéuticos está el dominio del análisis químico perfeccionado durante el siglo XIX. Lo anterior les permitió insertar la carrera de farmacia dentro de la Facultad de Ciencias Químicas - hoy Facultad de Química- y encabezar su administración durante varias décadas acuñando el modelo de enseñanza químico-farmacéutica que prevalece hoy en día en el país y que hermana a las licenciaturas de químico, químico farmacéutico biólogo, químico en alimentos e ingeniero químico, en tanto se imparten en un mismo recinto.

Cabe entonces preguntarse si en el futuro conviene deslindarse de las facultades de química y fundar recintos de farmacia y en qué términos se hará el deslinde, o si debemos caminar a una transformación gradual o súbita, completa o parcial, de fondo o de forma. El desconocimiento de los orígenes de la profesión conlleva el riesgo de comprometer la identidad profesional, ya de por sí mermada por el hecho de ser profesionales tripartitas cuya denominación no existe en el resto del mundo y por lo tanto son incomprendidos. En todo caso, la decisión sobre el futuro de la profesión deberá asumir el conocimiento $-\mathrm{O}$ desconocimiento- de la historia de la farmacia en México.

Referencias

Academia Farmacéutica de la Capital de la República (1846). Farmacopea Mexicana. México: Asociación Farmacéutica Mexicana (edición facsimilar 1997).

Aceves, P. (2011). La crisis de la farmacia mexicana, en J. Dosil y G. Sánchez (eds.), Continuidades y rupturas. Una historia tensa de la ciencia en México (pp. 278-311). México: UMSNH/UNAM.

Aceves, P. (1995). Hacia una farmacopea nacional: la primera farmacopea del México independiente, en P. Aceves (ed.), Farmacia, bistoria natural y química intercontinentales (pp.161-177). México: UAM-X.

Aceves, P. (1997). Ciencias químicas y médico-farmacéuticas en el siglo XIX mexicano. Revista Mexicana de Ciencias Farmacéuticas, 28(6), 27-31.

Aceves, P. (2008). Introducción al estudio de la química ó conocimientos preliminares para facilitar el estudio de la ciencia del Dr. Leopoldo Río de la Loza. México: Universidad Autónoma Metropolitana (edición facsimilar).

Aceves, P. y Martínez, S. (2010). Los farmacéuticos y los químicos mexicanos en la búsqueda de su identidad en los inicios del siglo $\mathrm{xx}$, en J. A. Chamizo (ed.), Historia y filosofía de la quimica: aportes para la enseñanza (pp.114-125). México: Siglo xxi.

Altamirano, F. (1903). Presupuesto para aparatos del Instituto Médico Nacional, México: Archivo General de la Nación GD 125/ IPBA/vol. 127/exp.29, 188 fojas.

Altamirano, F. (1905). Historia y objeto del Instituto Médico Nacional. México: Archivo General de la Nación GD 125/IPBA/vol. 128/exp.16, 8 fojas. 
Anderson, S. (2005). Making medicines a brief history of pharmacy and pharmaceutics. London: Pharmaceutical Press.

Annet, H. E. y Sen, H. (1921). Dosificación de la Codeína. La Farmacia, Nueva Serie, 33, 563-564.

Azuela, L. F. (1994). Positivismo, química y método experimental en la investigación biomédica mexicana a finales del siglo XIX, en P. Aceves (ed.), La quimica en Europa y América siglos XVIII y XIX (pp. 254-272). México: UAM-X.

Bertomeu, J. R. y Simon, J. (2012). Viejos objetos y nuevas perspectivas historiográficas: la cultura material de la ciencia en las aulas del siglo xix, en L. López-Ocón et al. (eds.), Aulas con memoria. Ciencia, educación y patrimonio en los institutos históricos de Madrid (1837-1936) (pp. 37-49). Madrid: Doce Calles.

Cervantes, V. (1889). Ensayo para la materia médica vegetal. El Estudio. México: Secretaría de Fomento.

De la Cal, A. (1832). Ensayo para la materia médica mexicana, arreglado por una comisión nombrada por la Academia Médico-Quirúrgica de esta capital. Puebla: Oficina del Hospital de San Pedro.

De los Ríos, J. L. (2011). Químicos y química. Ciudad de México: FCE/sep/Conacyt.

Dorvault, P. (1867). Repertorio universal de farmacia práctica o la oficina de farmacia. Madrid: Bailly-Bailliere.

Friedrich, C. (1996). The beginnings of the industrial era of pharmacy in Germany, en Pötzsch (ed.), The Pharmacy. Windows on History (pp.243-255). Basilea: Roche.

García, A., Bertomeu, J. R., Simó, J. (2005). Instrumentos y prácticas de enseñanza de las ciencias físicas y químicas en la Universidad de Valencia, durante el siglo XIx. Endoxa: Series Filosóficas, 19, 59-124.

Giral, C. (2015). Hacia la homologación del título de farmacia I: Panorama Actual.
Conferencia inaugural. I reunión hacia la Homologación del Título de Farmacia, I-Panorama Actual. México: AmeffarCOMAEFF.

Hinke, N. (2012). El Instituto Médico Nacional. La politica de las plantas y los laboratorios a fines del siglo XIX. México: UnAm/CINVESTAV.

Huerta, A. M. (2000). La primera materia médica del México independiente: influencias y procedencias, en P. Aceves (ed.), Tradiciones e intercambios cientificos: materia médica, farmacia y medicina (pp.301316). México: Uam-X.

Herrera, A. L. (1889). Diálisis química. Aplicaciones del sulfato de cal (tesis de licenciatura no publicada). México: Escuela Nacional de Medicina.

Heiduschka y Falul, A. (1921). Método para la determinación cuantitativa de cantidades muy pequeñas de morfina por vía colorimétrica, La Farmacia, 2(24), 392-393.

Ihde, A. J. (2015). The development of modern chemistry. New York: Dover Publications.

Livingstone, D. (2003). Putting science in its place. Geographies of scientific knowledge. London: University of Chicago Press.

Martínez, S., Aceves, P. y Morales, A. (2007). Una nueva identidad para los farmacéuticos. La sociedad mexicana en el cambio de siglo (1890-1919). Dynamis, 27, 263-285.

Morales, A. (2014). La búsqueda del sucedáneo: las investigaciones farmacológicas del chicalote (Argemone mexicana L.), en M. Ramos y F. León (eds.), Aportes recientes a la historia de la química en México

(pp.113-129). México: IQ/Ceich-unam. oms (Organización Mundial de la Salud) (2002). Promoción del uso racional de medicamentos: componentes centrales. OMS/ EDM/2002.3.

Ortiz, M. (2002). Las tesis de farmacia del siglo XIX mexicano. México: IQ/CNQFB/UAM-X.
Ortiz, M., Puerto, S., y Aceves, P. (2008). La reglamentación del ejercicio farmacéutico en México. Parte I (1841-1902). Revista Mexicana de Ciencias Farmacéuticas, 39(1), 12-19.

Ortiz, M. (2014a). La Legislación Farmacéutica en México: disposiciones sobre el ejercicio profesional, 1917-1973. Revista Mexicana de Ciencias Farmacéuticas, 45(1), 26-34.

Ortiz, M. (2014b). Preparación y uso de las drogas heroicas en México: 1846-1904, en M. Ramos y F. León (eds.), Aportes recientes a la historia de la química en México (pp.93111). México: IQ/Ceich-unam.

Ortiz, M. (2014c). Historia y legislación farmacéuticas: herramientas indispensables para describir el perfil del QFB. Enlace Quimico, 4(8), 8-13.

Pérez, R. (1885) La falsa belladona (tesis de licenciatura no publicada). Escuela Nacional de Medicina.

Pérez, F. (1905). De la esterilización en soluciones de clorhidrato de cocaína. La Farmacia, XIV(11), 243-251.

Río de la Loza, L. (2008). Introducción al estudio de la química o conocimientos preliminares para facilitar el estudio de la ciencia. México: UAM-X (edición facsimilar).

Schifter, L. (2014). Espiritu e identidad farmacéuticos. La construcción de la Farmacopea Mexicana (1846-2011). México: UAm-x.

Schifter, L. (2014). Las farmacopeas mexicanas en la construcción de la identidad nacional. Revista Mexicana de Ciencias Farmacéuticas, 45(2), 43-54.

Schifter, L., y Morales, A. (2012) La trayectoria de Francisco Río de la Loza en la Sección de Química Analítica del Instituto Médico Nacional. Revista Mexicana de Ciencias Farmacéuticas, 43(4), 69-78.

SFM (Sociedad Farmacéutica Mexicana) (1874). Nueva Farmacopea Mexicana. México: Imprenta de Ignacio Escalante 